\title{
Do working memory-driven attention shifts speed up visual awareness?
}

\author{
Yi Pan • Qiu-Ping Cheng
}

Published online: 12 August 2011

(C) Psychonomic Society, Inc. 2011

\begin{abstract}
Previous research has shown that content representations in working memory (WM) can bias attention in favor of matching stimuli in the scene. Using a visual priorentry procedure, we here investigate whether such WMdriven attention shifts can speed up the conscious awareness of memory-matching relative to memory-mismatching stimuli. Participants were asked to hold a color cue in WM and to subsequently perform a temporal order judgment (TOJ) task by reporting either of two different-colored circles (presented to the left and right of fixation with a variable temporal interval) as having the first onset. One of the two TOJ circles could match the memory cue in color. We found that awareness of the temporal order of the circle onsets was not affected by the contents of WM, even when participants were explicitly informed that one of the TOJ circles would always match the WM contents. The null effect of WM on TOJs was not due to an inability of the memory-matching item to capture attention, since response times to the target in a follow-up experiment were improved when it appeared at the location of the memory-matching item. The present findings suggest that WM-driven attention shifts cannot accelerate phenomenal awareness of matching stimuli in the visual field.
\end{abstract}

Keywords Working memory · Prior entry · Attention · Awareness

Attention is commonly assumed to facilitate processing of sensory input. According to the law of prior entry, "the object of attention comes to consciousness more quickly

Y. Pan $(\bowtie) \cdot$ Q.-P. Cheng

Department of Psychology, Hangzhou Normal University,

Hangzhou 310036, People's Republic of China

e-mail: panyirich@zju.edu.cn than the objects which we are not attending to" (Titchener, 1908 , p. 251). Converging evidence has recently accumulated suggesting that attention shifts from spatial precuing can accelerate the visual perceptual processing of stimuli appearing at cued, as opposed to uncued, locations (see Spence \& Parise, 2010, for a review). Previous research showing evidence for the accelerative effect of attention on visual perception has usually employed temporal order judgment (TOJ) tasks (e.g., Scharlau, 2004; Shore, Spence, \& Klein, 2001; Stelmach \& Herdman, 1991). For example, Shore et al. presented observers with two line segments (one vertical and one horizontal) with a variable stimulus onset asynchrony (SOA), one on the left side of fixation and one on the right side. Observers were required to report whether a horizontal or a vertical line segment was presented first. Before the onsets of the two line segments, there could be a spatial cue presented to the left or right of fixation. There were three cue conditions: In the verticalcued condition, the vertical stimulus was presented on the cued side, and the horizontal stimulus was presented on the uncued side. In the horizontal-cued condition, the horizontal stimulus was presented on the cued side and the vertical stimulus was presented on the uncued side. In the neutralcued condition, neither the horizontal nor the vertical stimulus was precued. The TOJ function for each of the three cue conditions was produced on the basis of proportions of responses indicating that the horizontal stimulus had the first onset at different SOAs. The results showed an evident horizontal shift in the TOJ function, suggesting that spatial precuing significantly influenced the TOJ task. The point of subjective simultaneity (PSS; i.e., the SOA between the two stimuli at which observers were equally likely to make either order judgment) shifted significantly as a function of the cue condition, and this was the case even when the role of observers' response bias 
was assessed, indicating that attention shifts from spatial precuing induce attended stimuli to be perceived prior to unattended stimuli.

Many models of visual attention suggest that the contents of working memory (WM) play an important role in guiding the spatial allocation of visual attention (e.g., Bundesen, 1990; Desimone \& Duncan, 1995; Duncan \& Humphreys, 1989; Wolfe, 1994). These models propose that a target template held in WM serves to bias attention in favor of matching stimuli in the scene, inducing memorymatching stimuli to have a privileged processing advantage over mismatching stimuli. In addition to such voluntary guidance of attention through WM, recent research has shown converging evidence that WM contents can guide attention even though there is no explicit intention for observers to voluntarily shift attention to memory-matching stimuli, suggesting that WM-driven attention shifts can occur in a relatively automatic fashion (see Pan, 2010; Soto, Hodsoll, Rotshtein, \& Humphreys, 2008, for recent reviews on this topic). The present study aimed to address whether WM-driven attention shifts can speed up visual awareness of stimuli matching the contents of WM.

Previous research has shown consistent evidence that WM-driven attention shifts can improve manual response times (RTs) to a search target when it appears at the location of the memory-matching item (e.g., Carlisle \& Woodman, 2011a; Downing, 2000; Soto, Heinke, Humphreys, \& Blanco, 2005). Moreover, several other studies have provided converging evidence showing that WM-driven attention can also influence perceptual processing (e.g., Huang \& Pashler, 2007; Pashler \& Shiu, 1999; Soto \& Humphreys, 2006; Soto, Wriglesworth, Balani, \& Humphreys, 2010). For example, a recent study using signal detection analysis by Soto et al. (2010) demonstrated that WM-driven attention shifts can enhance visual perception of stimuli matching the contents of WM. Observers were presented with an object cue that should be held in WM and subsequently had to identify a target presented within a colored shape that could match the cue. Perceptual sensitivity to the target was enhanced when it was presented within memory-matching shapes relative to when it appeared within mismatching shapes. This benefit was not due to bottom-up priming because there were minimal effects of the cue when it was merely attended and not kept in WM.

In the present study, we sought to explore whether the contents of WM can affect the temporal aspects of visual perceptual processing. Specifically, we asked whether WMdriven attention shifts can speed up conscious perception of stimuli matching the current contents of WM. Because there has been clear evidence for the accelerative effect of spatially cuing attention on awareness, we conjectured that WM-driven attention shifts might also accelerate visual awareness. We directly tested this hypothesis using a paradigm in which WM and TOJ tasks were combined. Here, participants were asked to hold a color cue in WM, followed by a TOJ task in which two different-colored circles were presented to the left and right of fixation with a variable SOA. One of the two TOJ circles could match the memory cue in color. If WM-driven attention shifts can accelerate perception of memory-matching stimuli, the TOJ response function and the PSS should shift significantly as a function of whether the left circle, the right circle, or neither circle matched the contents of WM; otherwise, TOJ response functions of different matching conditions should overlap, and the differences in the PSS between those conditions should not be significant.

\section{Experiment 1}

Experiment 1 assessed whether automatic guidance of attention through WM can influence TOJs. Participants were required to hold a color cue in WM and to subsequently perform a TOJ task by reporting which of the two circles (one on the left side of fixation and one on the right side) had the first onset. The circles matched the color held in WM on only half of the trials, providing no explicit incentive for participants to deliberately search for the memory-matching circles. More importantly, in order to reduce the influence of response bias, we used a methodology in which memory cues and TOJ dimensions were orthogonal (cf. Shore et al., 2001). Specifically, participants were instructed to memorize colors while they reported whether the left circle or the right circle occurred first. Participants were informed that the colors of the circles were irrelevant to the tasks.

\section{Method}

Participants A group of 11 naive students at the Hangzhou Normal University participated for partial course credit. All of them reported having normal or corrected-to-normal visual acuity and normal color vision.

Stimuli The stimuli were presented on a 17 -in. CRT monitor with a resolution of $1,024 \times 768$ pixels and an $85-\mathrm{Hz}$ refresh rate. The visual stimuli were colored diamonds $\left(\right.$ each $3^{\circ} \times 3^{\circ}$ ) and circles $\left(\right.$ each $3^{\circ} \times 3^{\circ}$ ). The color of each shape was chosen randomly from a set of five colors (red, green, blue, yellow, and cyan). All stimuli were presented on a gray background at a viewing distance of $57 \mathrm{~cm}$.

Procedure and design Participants initiated each trial by pressing the space bar. Each trial began with the display of 
a white central fixation cross $\left(0.2^{\circ} \times 0.2^{\circ}\right)$ for $1,000 \mathrm{~ms}$. Participants were asked to fixate on the cross while allocating attention equally across the display. Then, a colored diamond was presented at the center of the screen for $1,000 \mathrm{~ms}$. Here, participants were instructed to memorize the color of the diamond and to keep it in mind throughout the entire trial. After a delay of $500 \mathrm{~ms}$, an onset of one colored circle occurred on the left or right of fixation. Following a variable $\operatorname{SOA}(0,12,24,48$, or $60 \mathrm{~ms}$ ), the second colored circle appeared at the opposite location relative to the first (i.e., if the first circle appeared on the left of fixation, the second circle appeared on the right of fixation, and vice versa). Each of the two circles was centered $3^{\circ}$ from fixation and remained visible on the screen until response. Participants were instructed to make unspeeded TOJs regarding which of the two circles appeared first by pressing the left button of the mouse for the left circle or the right button for the right circle. A memory test followed $500 \mathrm{~ms}$ after a response was made. Here, a diamond appeared at the center of the screen and participants were asked to indicate whether it had the same color as the memory cue or a different color by pressing one of the two buttons (see Fig. 1).

The memory test item and the memory cue shared the same color on half of the trials, and they had different colors on the other half. The two circles were always different from each other in color on each trial. Participants were never asked to report the color of a circle, but simply to indicate the spatial location of the circle that appeared first. According to the relationship between the memory cue and the two circles, there were three different cue conditions. In the left-cued condition, the color of the left circle matched the color of the memory cue. In the right-cued condition, the color of the right circle matched the color of the memory cue. The leftand right-cued conditions each occurred on $25 \%$ of trials. The remaining $50 \%$ of trials were the neutral-cued condition, in which neither of the colors of the left and right circles matched the color of the memory cue. These probabilities for the three cue conditions were the same on each SOA. The three cue conditions were not mentioned to participants and were randomized across trials. This protocol provided participants with no explicit incentive to deliberately search for the memory-matching circle in the TOJ task. Both the left and the right circle were equally likely to have the first onset on each nonzero SOA. Trial order was randomized across the factors of first-onset circle and SOA.

\section{Results and discussion}

Errors on the memory task averaged $6 \%$ and did not vary significantly across conditions (see Table 1). For each of the three cue conditions, we calculated the proportion of responses indicating that the left circle was perceived first at a given SOA. Figure 2 shows these data averaged across participants. Negative SOAs refer to when the left circle was presented first, whereas positive SOAs refer to when the right circle was presented first. As is evident in Fig. 2, the three psychometric functions overlapped almost completely. This observation was confirmed by a 3 (cue condition: left, neutral, and right cued) $\times 9$ (SOA: $-60,-48,-24,-12,0,12,24,48$, and $60 \mathrm{~ms})$ ANOVA. The main effect of SOA was significant, $F(8,80)=226.331, p<.0001, \eta^{2}=.958$, indicating that participants were complying with the TOJ task and that their ability to discriminate the temporal order of the onsets of the two circles was maximal at longer SOAs, while it was minimal at shorter SOAs (i.e., points near the PSS). Neither the main effect of cue condition $(F<1)$ nor the interaction between the two factors $(F<1.02)$ was significant. Also, the PSSs did not differ significantly between the left-cued condition $(M=7.7 \mathrm{~ms}, S E=2.8)$, the neutral-cued condition $(M=5.5 \mathrm{~ms}, S E=3.2)$, and the right-cued condition $(M=6.1 \mathrm{~ms}, S E=3.4), F<1$. These findings indicate that the contents of WM did not influence TOJs. If the contents of WM had accelerated awareness of matching stimuli relative to mismatching stimuli, then there should have been an apparent horizontal shift in the TOJ function and in the PSS between those different cue conditions. This was clearly not the case, indicating that the contents of WM did not show visual prior entry.
Fig. 1 Schematic illustration of the trial sequence and example stimuli used in Experiment 1

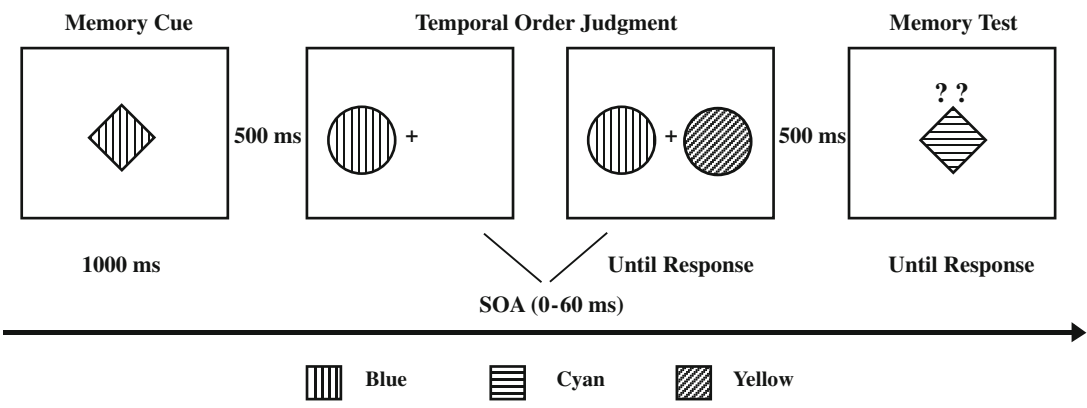


Table 1 Mean proportions of error memory responses as a function of cue condition and SOA between the left and right circles across Experiments 1 and 3

\begin{tabular}{llllllllll}
\hline \multicolumn{7}{l}{ SOA (ms) } \\
\cline { 2 - 9 } & -60 & -48 & -24 & -12 & 0 & 12 & 24 & 48 & 60 \\
\hline Experiment 1 & & & & & & & & & \\
Left cued & .069 & .051 & .062 & .053 & .062 & .061 & .059 & .052 & .054 \\
Neutral cued & .071 & .058 & .069 & .059 & .061 & .066 & .062 & .059 & .060 \\
Right cued & .059 & .062 & .059 & .068 & .057 & .053 & .066 & .062 & .052 \\
Experiment 3 & & & & & & & & & \\
Left cued & .037 & .046 & .033 & .047 & .048 & .056 & .034 & .056 & .036 \\
Right cued & .034 & .044 & .056 & .044 & .043 & .034 & .043 & .044 & .033 \\
\hline
\end{tabular}

\section{Experiment 2}

Previous research has demonstrated boundary constraints on the effects of WM on attention (e.g., Han \& Kim, 2009; Olivers, 2009; Pan \& Soto, 2010; Woodman \& Luck, 2007). Therefore, it is possible that the null effect of WM on TOJs in Experiment 1 was due to the inability of the memory-matching item to draw attention in the present task paradigm. We addressed this possibility by directly testing whether the circle that matched the color held in WM could capture attention in Experiment 2.

\section{Method}

The method was similar to that of Experiment 1, with the following exceptions. A new group of 9 volunteers from the same pool participated in this experiment. At $500 \mathrm{~ms}$ after the offset of the memory cue, two prime stimuli

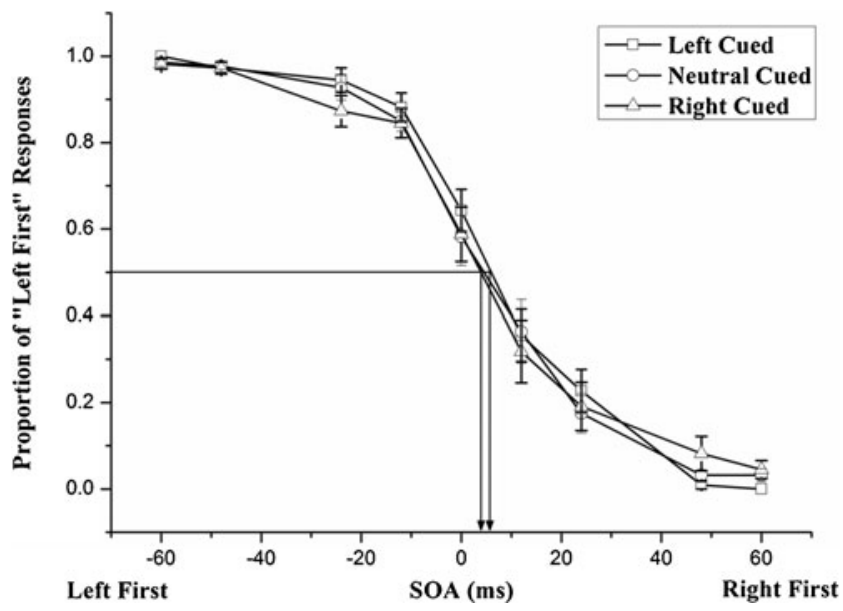

Fig. 2 The average proportions of "left first" responses as a function of the cue condition and the stimulus onset asynchrony (SOA) between left and right circles in Experiment 1. Negative SOAs indicate that the left circle was presented first, whereas positive SOAs indicate that the right circle was presented first. The horizontal line indicates the point at which $50 \%$ of the responses were "left first." The arrows indicate the point of subjective simultaneity for each of the cue conditions. Error bars represent standard errors (colored circles) were simultaneously presented for $200 \mathrm{~ms}$ to the left and right of fixation, separated by $6^{\circ}$ from center to center. After a 40-ms delay, a search array consisting of two black outlined squares (each $0.6^{\circ} \times 0.6^{\circ}$ ) with a gap at the top, the bottom, the left, or the right side was displayed until a response was made by participants. The two squares appeared at the centers of the locations previously occupied by the two circles. The search array contained a target and a distractor. The target had a gap on the top or the bottom, and the distractor had a gap on the left or the right (see Fig. 3). Participants were instructed to make a speeded response to the orientation (top vs. bottom) of the target by pressing one of two different keys with the right hand. A memory test followed $500 \mathrm{~ms}$ after the response to the search task. Participants were required to indicate whether the memory test item did or did not have the same color as the memory cue by pressing one of the two keys with the left hand. There were three types of trials. On valid trials, one of the two circles shared its color with the memory cue, and the search target appeared at the location of the memory-matching circle. On invalid trials, one of the two circles matched the color of the memory cue, and the target appeared at the location of the mismatching circle. On neutral trials, neither of the two circles matched the color of the memory cue. All told, 25\%, 25\%, and $50 \%$ of the total number of trials were of the valid, invalid, and neutral types, respectively.

\section{Results and discussion}

Errors averaged $0.4 \%$ on the search task and $4.4 \%$ on the memory task (see Table 2). A one-way ANOVA with trial type as a factor performed on both search and memory errors showed no significant effects $(p s>.266)$. Analyses of RTs in the search task included only trials on which participants were accurate in both the search and memory tasks. To account for outliers, we calculated median RTs for each participant on each of the three types of trials. The main effect of trial type on median RTs was significant, $F(2$, 16) $=4.353, p=.035$ (Greenhouse-Geisser corrected), $\eta^{2}=$ 
Fig. 3 Schematic illustration of the trial sequence and example stimuli used in Experiment 2

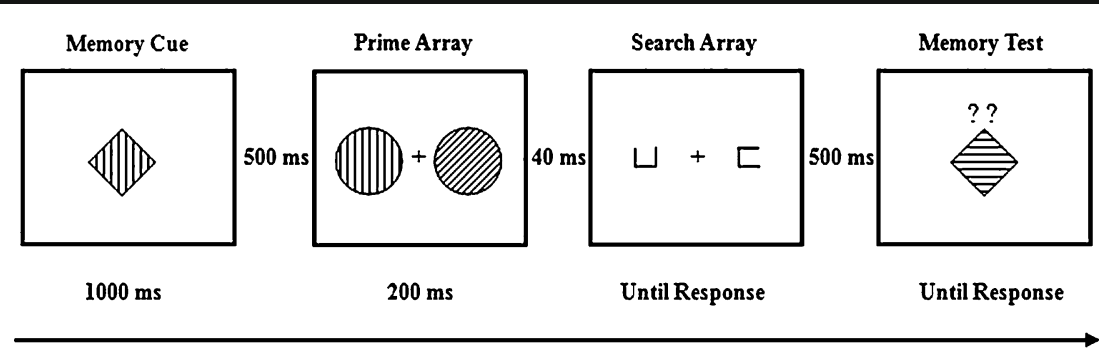

IIIII Blue 国 cyan Yellow
.352 , with faster performance on valid trials $(M=634 \mathrm{~ms}$, $S E=29)$ than on invalid $(M=680 \mathrm{~ms}, S E=33)$ and neutral $(M=668 \mathrm{~ms}, S E=30)$ trials. This suggests that attention was automatically deployed to the circle that matched the color held in WM, speeding up target discrimination when it appeared at the location of the memory-matching circle. Thus, we cannot account for the result that WM contents did not show prior entry in Experiment 1 by the inability of the memory-matching stimuli to capture attention.

\section{Experiment 3}

The prior experiments showed that WM contents can automatically guide attention to the memory-matching stimuli in the field, but that such involuntary shifts of attention via WM do not induce memory-matching stimuli to be perceived earlier, as compared to mismatching stimuli. Experiment 3 further tested whether voluntary shifts of attention based on the contents of WM can make memorymatching stimuli show prior entry. Here, one of the two objects in the TOJ task always matched the color held in WM, and thus participants could deliberately search for the memory-matching item in order to help their performance.

\section{Method}

This experiment was identical to Experiment 1, with the following exceptions. A group of 12 new volunteers from the same pool took part here. These participants were explicitly informed that one of the two TOJ circles would always match the color of the memory cue on each trial.

Table 2 Mean proportions of error responses in the search and memory tasks as a function of cue validity across Experiments 2 and 4

\begin{tabular}{llllll}
\hline & \multicolumn{3}{c}{ Experiment 2} & & \multicolumn{2}{l}{ Experiment 4} \\
\cline { 2 - 3 } \cline { 5 - 5 } & Search Task & Memory Task & & Search Task & Memory Task \\
\hline Valid & .006 & .046 & .017 & .047 \\
Neutral & .003 & .052 & & .061 \\
Invalid & .006 & .038 & .022 & .06 \\
\hline
\end{tabular}

Thus, there were left- and right-cued trials (which occurred with equal probability), but no neutral-cued trials. This protocol provided participants with explicit incentive to deliberately search for the memory-matching circle in the TOJ task.

\section{Results and discussion}

Errors on the memory task averaged $4 \%$ and did not vary significantly across conditions (see Table 1). Figure 4 shows the average proportion of responses indicating that the left circle was perceived first at a given SOA for each cue condition, with negative SOAs indicating that the left circle was presented first and positive SOAs indicating that the right circle was presented first. As in Experiment 1, the two psychometric functions overlapped. This was confirmed by a 2 (cue condition: left cued and right cued) $\times 9$ (SOA: $-60,-48,-24,-12,0,12,24,48$, and $60 \mathrm{~ms}$ ) ANOVA, which only yielded a significant main effect of SOA, $F(8,88)=232.653, p<.0001, \eta^{2}=.955$. Neither the main effect of cue condition $(F<1)$ nor the interaction

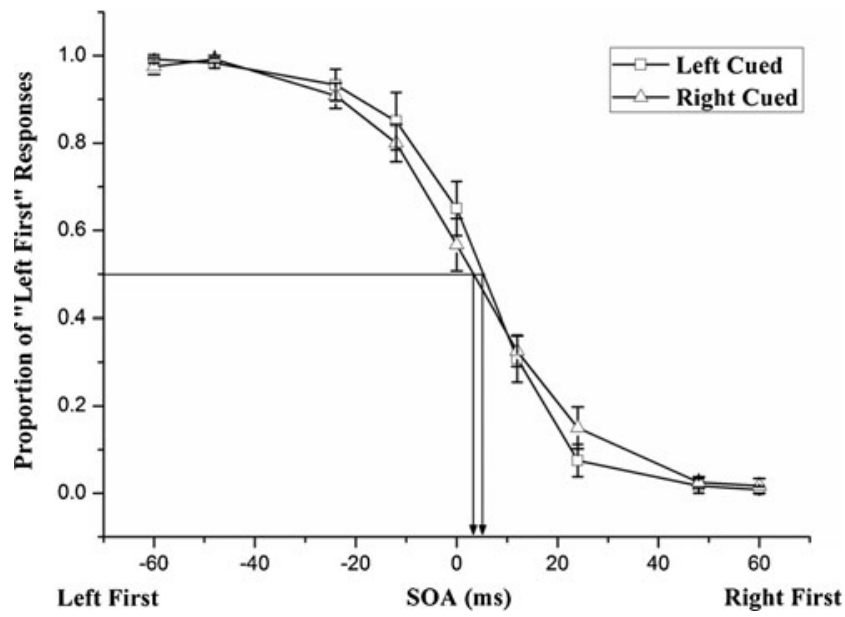

Fig. 4 The average proportions of "left first" responses as a function of the cue condition and the stimulus onset asynchrony (SOA) between left and right circles in Experiment 3. Negative SOAs indicate that the left circle was presented first, whereas positive SOAs indicate that the right circle was presented first. The horizontal line indicates the point at which $50 \%$ of the responses were "left first." The arrows indicate the point of subjective simultaneity for each of the cue conditions. Error bars represent standard errors 
between the two factors $(F<1.08)$ approached significance. Also, the PSSs did not differ significantly between the leftcued condition $(M=6.5 \mathrm{~ms}, S E=3.2 \mathrm{~ms})$ and the rightcued condition $(M=5 \mathrm{~ms}, S E=2.8 \mathrm{~ms}), F<1$. Again, there was no evidence for the contents of WM showing visual prior entry.

\section{Experiment 4}

Experiment 4 aimed to further rule out the possibility that the null effect of WM on TOJs in Experiment 3 was due to no attention capture by WM.

Method

This experiment was identical to Experiment 2, with the following exceptions. A new group of 9 volunteers from the same pool participated. They were informed that one of the two circles would always match the color held in WM on each trial. The search target appeared at the location of the matching circle on half of the trials (valid trials) and at the location of the mismatching circle on the other half (invalid trials).

\section{Results and discussion}

Errors averaged $1.9 \%$ on the search task and $5.4 \%$ on the memory task (see Table 2). There was no significant difference between valid and invalid trials on either search or memory errors $(p s>.325)$. A one-way ANOVA on median RTs of the correct responses showed faster search performance on valid $(M=602 \mathrm{~ms}, S E=24)$ than on invalid $(M=642 \mathrm{~ms}, S E=31)$ trials, $F(1,8)=$ $7.998, p=.022, \eta^{2}=.5$, suggesting that the contents of WM did guide attention to the memory-matching items. Therefore, we cannot account for the lack of evidence for accelerated perception of the memory-matching items in Experiment 3 by a lack of attention shifts driven by WM.

\section{Experiment 5}

The aim of the final experiment was to test whether the null results in Experiments 1 and 3 were due to a lack of power induced by the TOJ objects used here. It is possible that, for some unknown reason, the prior-entry effect could not be observed with the present colored circles, even if traditional exogenous spatial attention were used (Shore et al., 2001). To address this concern, in Experiment 5 we examined the TOJ function and the PSS with the same colored circles we had used in prior experiments, but now attention to the TOJ objects was elicited exogenously by a task-irrelevant cue consisting of a peripheral flash (Jonides, 1981). If null results were still observed with exogenous cuing of attention, this would favor a lack-of-power account; otherwise, we could not account for the lack of an effect of WM on TOJs by a lack of power.

Method

This experiment was similar to Experiment 1, except that no memory task was used here. Following the initial fixation display, a cue consisting of a black dot $\left(0.9^{\circ} \times\right.$ $0.9^{\circ}$ ) was flashed for $59 \mathrm{~ms}$. This cue could appear either at fixation (neutral-cued condition) or just above $\left(1.5^{\circ}\right.$ vertically) one of the two peripheral positions ( $3^{\circ}$ to the left or right of fixation) where the TOJ objects would appear $50 \mathrm{~ms}$ later (left- and right-cued conditions). A new group of 9 volunteers from the same pool participated. They were informed that the cue was spatially uninformative about which of the two TOJ objects would be presented first and that they had to maintain their gaze at fixation throughout the trial.

\section{Results and discussion}

We calculated the proportions of responses indicating that the left circle was perceived first as a function of SOA and cue condition. The results depicted in Fig. 5 show apparent shifts in the TOJ function between the three cue conditions, with a horizontal shift to the left for the right-cued

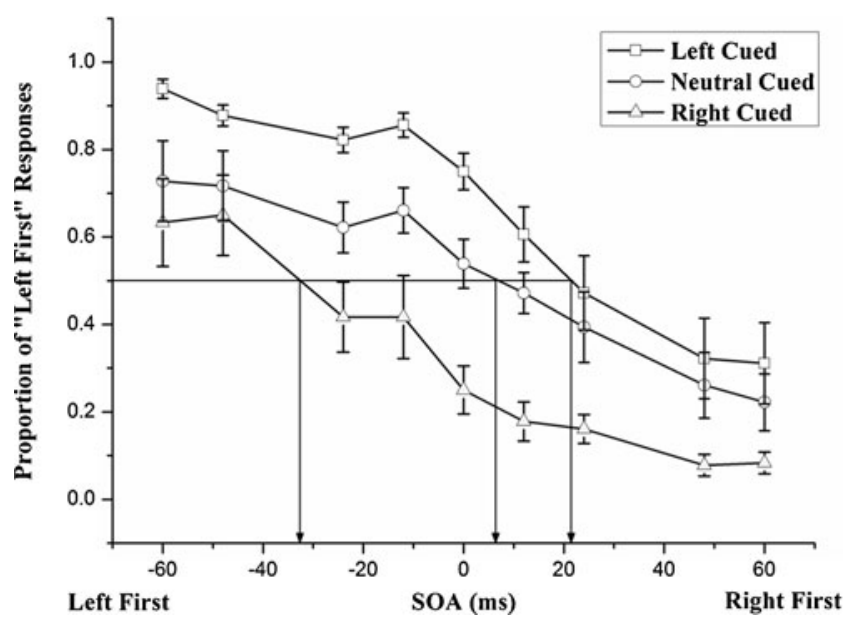

Fig. 5 The average proportions of "left first" responses as a function of the cue condition and the stimulus onset asynchrony (SOA) between left and right circles in Experiment 5. Negative SOAs indicate that the left circle was presented first, whereas positive SOAs indicate that the right circle was presented first. The horizontal line indicates the point at which $50 \%$ of the responses were "left first." The arrows indicate the point of subjective simultaneity for each of the cue conditions. Error bars represent standard errors 
condition and to the right for the left-cued condition, as compared to the neutral-cued baseline. Such an evident change in TOJs as a function of exogenous cuing was confirmed by a 3 (cue condition: left, neutral, and right cued) $\times 9$ (SOA: $-60,-48,-24,-12,0,12,24,48$, and $60 \mathrm{~ms}$ ) ANOVA, which yielded a significant main effect of cue condition, $F(2,16)=11.108, p=.006$ (Greenhouse-Geisser corrected), $\eta^{2}=.581$. The main effect of SOA was also significant, $F(8,64)=26.598, p<.0001$, $\eta^{2}=.769$, indicating that participants were complying with the TOJ task. The interaction between the two factors approached significance, $F(16,128)=2.89, p=.061$ (Greenhouse-Geisser corrected), $\eta^{2}=.265$, which suggests that the effect of exogenous cuing on TOJs tended to be reduced at the longer SOAs (e.g., 48 and $60 \mathrm{~ms}$ ). Also, the PSSs varied significantly as a function of the cue condition, $F(2,16)=23.623, p=.001$ (GreenhouseGeisser corrected), $\eta^{2}=.747$. The present results confirmed those of Shore et al. (2001), suggesting that exogenous cuing can significantly influence TOJs, probably via both accelerated perception and a response bias for those stimuli appearing at cued locations. Thus, the reason why we did not find evidence for WM effects on TOJs in prior experiments is unlikely to be a lack of power due to the colored circles we used.

\section{General discussion}

The close connection between WM and attention has been repeatedly proposed and demonstrated. Converging evidence has recently accumulated suggesting that the contents of WM can guide attention in favor of memory-matching stimuli within the visual field (for recent reviews, see Pan, 2010; Soto et al., 2008). The present study aimed to explore whether such WM-driven attention shifts can accelerate visual awareness. Since there has been clear evidence showing the accelerative effects of spatially cuing attention on perception (e.g., Scharlau, 2004; Shore et al., 2001; Stelmach \& Herdman, 1991), one might expect that WMdriven attention shifts would also accelerate visual awareness of memory-matching stimuli relative to mismatching stimuli. However, we found no evidence for significant modulation of TOJs by the contents of WM, even when observers were explicitly informed that one of the two TOJ stimuli would always match the information in WM. The PSS also did not shift significantly across different cue conditions. These null results cannot be attributed (1) to the inability of the memory-matching stimuli in the present study to capture attention, because RTs to a search target were improved when it appeared at the location of a memory-matching item, indicating that attention had actually shifted to the memory-matching stimulus; or (2) to a lack of power due to the colored shapes used here, because the visual prior-entry effect emerged when attention was directed to these stimuli using an exogenous spatial precue. Although prudence is always necessary when drawing conclusions from null results, the present findings indicate that WM-driven attention shifts do not speed up visual awareness of stimuli matching the contents of WM, at least under the experimental conditions tested here. Because the memory cues in our study never appeared at the same location as a TOJ item and were maintained in WM throughout the trial, effects of bottom-up spatial attention shifts via exogenous cuing were eliminated, and we focused only on whether top-down attention shifts driven by WM could influence TOJs. The present results extend previous research regarding the prior-entry effect (see Spence \& Parise, 2010, for a review) by providing an important boundary condition on the accelerative effects of attention on perception: Feature-based WM-driven attention may not exert a significant impact on accelerating visual awareness.

Our results may seem to be at odds with those of Stolz (1999), who asked observers to report which of two unrelated TOJ words appeared first. When one word was semantically primed by a masked prime word briefly presented at the center of the screen at the beginning of a trial, the semantically primed word was reported to occur first more often than the unprimed word at short SOAs between the two TOJ words. Stolz argued that semantic priming affected TOJs via perceptual prior entry for the primed words. However, it could also be that her effect of priming on TOJs was due to a response bias. That is, when observers were uncertain about the temporal order at very short SOAs between the TOJ words, they could be biased to indicate that the primed word was the first onset. Indeed, recent work by Burnham, Neely, and O'Connor (2006) has clearly demonstrated the role of response bias in the influence of word priming on TOJs, although there was indeed a prior-entry effect for the primed word relative to the unprimed one. Moreover, the present null results might have important implications for understanding the underlying mechanism of the word-priming effects on TOJs. Note that the present research differed from that of Stolz (1999) and Burnham et al. (2006) in an important way: The experimental stimuli used here were colored shapes rather than words, and an exact physical feature was primed, not the lexical information of a TOJ item. Our results indicate that merely priming the physical feature of an object cannot influence TOJs via accelerated perception or a response bias for the primed item. We suggest that word priming affects TOJs primarily via a lexical mechanism, whereas mere physical identity priming without lexical priming does not influence TOJs, even though the prime identity is actively held "online" in WM. 
How can we explain the lack of prior entry into visual awareness for the contents of WM? We suggest the following three possibilities: (1) Some evidence has shown that endogenous cuing of attention exerts a much smaller accelerative effect on perception than does exogenous cuing of attention (Shore et al., 2001). It seems that WM-driven attention is similar to endogenous (i.e., top-down) attention, not to exogenous (i.e., bottom-up) attention. Thus, it is possible that WM-driven attention might occur in a more top-down fashion than has previous endogenous cuing of attention, leading to the inability for WM-driven attention to accelerate perception. (2) The effects of WM on visual perception, if any, are probably generated via the enhanced perceptual representations of memory-matching stimuli in the visual field (Chelazzi, Miller, Duncan, \& Desimone, 1993, 2001; Soto \& Humphreys, 2006; Soto et al., 2010). Nevertheless, the enhanced perception of a stimulus does not necessarily lead to accelerated awareness of it. Thus, a stimulus matching the current contents of WM, which has a strengthened representation via top-down modulation from WM, might not reach awareness earlier than a mismatching one when they appear at the same moment in the visual field. (3) Converging evidence has recently accumulated suggesting that the influence of WM on perceptual processing may be subject to cognitive control (e.g., Carlisle \& Woodman, 2011a, b; Han \& Kim, 2009; Pan \& Soto, 2010; Woodman \& Luck, 2007). Thus, in the present study the TOJ task might require more cognitive control than the visual search task, rendering more severe constraints of the effect of WM on TOJs than on search RTs. If so, this would mean that the TOJ task is not a sensitive paradigm for assessing the potential accelerative effect of WM on perception, and that future research using a more elegant paradigm is needed.

Our negative findings are consistent with those reported by Turatto, Vescovi, and Valsecchi (2008). They showed that WM-driven attention shifts do not alter the perceived motion speed of memory-matching stimuli. However, because a stimulus has many different properties, and the perceptual mechanisms of those stimulus properties are also very different from one another, the null effects of WM on perceptual experiences of temporal order and motion speed would not necessarily generalize to other aspects of conscious processing. A recent study in our lab, for instance, showed that the contents of WM can affect the perceived duration of a stimulus (Pan \& Luo, 2011). During participants' WM retention interval, two colored circles were presented in succession at the center of the screen for different durations, with one circle matching the color of the memory cue and the other mismatching the cue. Immediately after the offset of the second circle, participants were asked to make an unspeeded judgment regarding which of the two circles was presented for a longer (or shorter) duration. The results showed more duration judgment errors when the memory-matching circle had a shorter duration than the mismatching one, as compared to when the memorymatching circle had a longer duration than the mismatching one. This effect was not due to the mechanism of bottom-up perceptual priming, because mere exposure to the color cue without WM processing actually led to a reversed pattern of results. These findings suggest that active maintenance of an object in WM prolongs the perceived duration of matching stimuli in the visual field. Considering the oft-repeated view that "the more intense a stimulation, the longer the duration perceived" (Fraisse, 1978 , p. 220), the effect of WM on subjective duration may be generated through the enhanced perceptual representations of stimuli that match the contents of WM (Soto \& Humphreys, 2006; Soto et al., 2010).

In summary, the present results suggest that WM-driven attention shifts can accelerate manual responses but not phenomenal awareness. This corroborates the view of the dissociation between attention and awareness (Lamme, 2003). In line with the evidence suggesting that allocation of attention to an object may not always be sufficient to make that object reach awareness (e.g., Kentridge, Heywood, \& Weiskrantz, 2004; Kentridge, Nijboer, \& Heywood, 2008; Woodman \& Luck, 2003), our findings imply that WM-driven attention also may not be sufficient to allow stimuli matching the contents of WM to be perceived earlier than mismatching stimuli.

Author note We thank Bradley Gibson, Liqiang Huang, Geoff Woodman, and Scott Watter for their valuable comments and suggestions. This research was supported by grants from the National Natural Science Foundation of China (31000462), the Zhejiang Provincial Social Science Foundation (10CGJY11YB), and the Qinshen Research Project of the Hangzhou Normal University.

\section{References}

Bundesen, C. (1990). A theory of visual attention. Psychological Review, 97, 523-547. doi:10.1037/0033-295X.97.4.523

Burnham, B. R., Neely, J. H., \& O'Connor, P. A. (2006). Priming effects on temporal order judgments about words: Perceived temporal priority or response bias? Psychonomic Bulletin \& Review, 13, 429-433. doi:10.3758/BF03193865

Carlisle, N. B., \& Woodman, G. F. (2011a). Automatic and strategic effects in the guidance of attention by working memory representations. Acta Psychologica, 137, 217-225. doi:10.1016/ j.actpsy.2010.06.012

Carlisle, N. B., \& Woodman, G. F. (2011b). When memory is not enough: Electrophysiological evidence for goal-dependent use of working memory representations in guiding visual attention. Journal of Cognitive Neuroscience. doi:10.1162/ jocn.2011.21602 
Chelazzi, L., Miller, E. K., Duncan, J., \& Desimone, R. (1993). A neural basis for visual search in inferior temporal cortex. Nature, $363,345-347$.

Chelazzi, L., Miller, E. K., Duncan, J., \& Desimone, R. (2001). Responses of neurons in macaque area V4 during memoryguided visual search. Cerebral Cortex, 2001(11), 761-772.

Desimone, R., \& Duncan, J. (1995). Neural mechanisms of selective visual attention. Annual Review of Neuroscience, 18, 193-222. doi:10.1146/annurev.ne.18.030195.001205

Downing, P. E. (2000). Interactions between visual working memory and selective attention. Psychological Science, 11, 467-473. doi:10.1111/1467-9280.00290

Duncan, J., \& Humphreys, G. W. (1989). Visual search and stimulus similarity. Psychological Review, 96, 433-458.

Fraisse, P. (1978). Time and rhythm perception. In E. C. Carterette \& M. P. Freidman (Eds.), Handbook of perception, Vol. VIII: Perceptual coding (pp. 203-247). New York: Academic Press.

Han, S. W., \& Kim, M.-S. (2009). Do the contents of working memory capture attention? Yes, but cognitive control matters. Journal of Experimental Psychology. Human Perception and Performance, $35,1292-1302$.

Huang, L., \& Pashler, H. (2007). Working memory and the guidance of visual attention: Consonance-driven orienting. Psychonomic Bulletin \& Review, 14, 148-153. doi:10.3758/BF03194042

Jonides, J. (1981). Voluntary versus automatic control over the mind's eye's movement. In J. Long \& A. Baddeley (Eds.), Attention and performance IX (pp. 187-203). Hillsdale, NJ: Erlbaum.

Kentridge, R. W., Heywood, C. A., \& Weiskrantz, L. (2004). Spatial attention speeds discrimination without awareness in blindsight. Neuropsychologia, 42, 831-835.

Kentridge, R. W., Nijboer, T. C. W., \& Heywood, C. A. (2008). Attended but unseen: Visual attention is not sufficient for visual awareness. Neuropsychologia, 46, 864-869.

Lamme, V. A. F. (2003). Why visual attention and awareness are different. Trends in Cognitive Sciences, 7, 12-18. doi:10.1016/ S1364-6613(02)00013-X

Olivers, C. N. L. (2009). What drives memory-driven attentional capture? The effects of memory type, display type, and search type. Journal of Experimental Psychology. Human Perception and Performance, 35, 1275-1291.

Pan, Y. (2010). Content-based working memory-driven visual attention [in Chinese]. Advances in Psychological Science, $18,210-219$.

Pan, Y., \& Luo, Q.-Y. (2011). Working memory modulates the perception of time. Manuscript submitted for publication.

Pan, Y., \& Soto, D. (2010). The modulation of perceptual selection by working memory is dependent on the focus of spatial attention. Vision Research, 50, 1437-1444.
Pashler, H., \& Shiu, L.-P. (1999). Do images involuntarily trigger search? A test of Pillsbury's hypothesis. Psychonomic Bulletin \& Review, 6, 445-448. doi:10.3758/BF03210833

Scharlau, I. (2004). Evidence against response bias in temporal order tasks with attention manipulation by masked primes. Psychological Research, 68, 224-236. doi:10.1007/s00426-003-0135-8

Shore, D. I., Spence, C., \& Klein, R. M. (2001). Visual prior entry. Psychological Science, 12, 205-212.

Soto, D., Heinke, D., Humphreys, G. W., \& Blanco, M. J. (2005). Early, involuntary top-down guidance of attention from working memory. Journal of Experimental Psychology. Human Perception and Performance, 31, 248-261.

Soto, D., Hodsoll, J., Rotshtein, P., \& Humphreys, G. W. (2008). Automatic guidance of attention from working memory. Trends in Cognitive Sciences, 12, 342-348. doi:10.1016/j.tics.2008.05.007

Soto, D., \& Humphreys, G. W. (2006). Seeing the content of the mind: Enhanced awareness through working memory in patients with visual extinction. Proceedings of the National Academy of Sciences, 103, 4789-4792.

Soto, D., Wriglesworth, A., Balani, A. B., \& Humphreys, G. W. (2010). Working memory enhances visual perception: Evidence from single detection analysis. Journal of Experimental Psychology. Learning, Memory, and Cognition, 36, 441-456. doi:10.1037/ a0018686

Spence, C., \& Parise, C. (2010). Prior-entry: A review. Consciousness and Cognition, 19, 364-379. doi:10.1016/j.concog.2009.12.001

Stelmach, L. B., \& Herdman, C. M. (1991). Directed attention and perception of temporal order. Journal of Experimental Psychology. Human Perception and Performance, 17, 539-550.

Stolz, J. A. (1999). Word recognition and temporal order judgments: Semantics turns back the clock. Canadian Journal of Experimental Psychology, 53, 316-322. doi:10.1037/h0087319

Titchener, E. (1908). Lectures on the elementary psychology of feeling and attention. New York: Macmillan.

Turatto, M., Vescovi, M., \& Valsecchi, M. (2008). On altering motion perception via working memory-based attention shifts. Journal of Vision, 8(5), 11:1-13. doi:10.1167/8.5.11

Wolfe, J. M. (1994). Guided Search 2.0: A revised model of visual search. Psychonomic Bulletin \& Review, 1, 202-238. doi:10.3758/BF03200774

Woodman, G. F., \& Luck, S. J. (2003). Dissociations among attention, perception, and awareness during object-substitution masking. Psychological Science, 14, 605-611. doi:10.1046/j.09567976.2003.psci 1472.x

Woodman, G. F., \& Luck, S. J. (2007). Do the contents of visual working memory automatically influence attentional selection during visual search? Journal of Experimental Psychology. Human Perception and Performance, 33, 363-377. doi:10.1037/0096-1523.33.2.363 